\title{
Pioneers in Biomedical Optics: Special Section Honoring Professor Tayyaba Hasan
}

Professor Tayyaba Hasan is one of the pioneers in photodynamic therapy worldwide, having developed landmark discoveries in both basic and translational biomedicine. Her career hallmarks have been those of being willing to try new ideas, working with one foot in basic research and one foot in translational medicine, and an eagerness to work with and mentor developing researchers. Her achievements were acknowledged by the National Institutes of Health by giving her their Bench to Bedside Award in 2009. Her long career continues today without any sign of stopping, as she currently runs two major activities at the Massachusetts General Hospital (MGH). In her founding academic institution, she is a professor of dermatology at Harvard Medical School and a professor of health sciences and technology in the Harvard-MIT Division; in yet another capacity she is director of the Office for Research Career Development. She has served as a member of many review boards both internally with $\mathrm{MGH} /$ Harvard and externally for other organizations, and this past year she was awarded the William Silen Lifetime Achievement Award for Mentoring by the Harvard Medical School. She has chaired most major conferences in the field of photomedicine and biomedical optics at one time or another, and she has spawned the careers of countless scientists in the United States and abroad.

Prof. Hasan launched her scientific career in her home country of Pakistan, at the University of Karachi, where she obtained her BS in chemistry and MS in organic chemistry. She then moved onto the University of Islamabad, Pakistan, for an MPhil in physical chemistry. From there she initiated what turned out to be her permanent migration to the United States by entering a $\mathrm{PhD}$ program at the University of Arkansas in physical organic chemistry. She completed postdoctoral training at the University of Pennsylvania in chemistry,

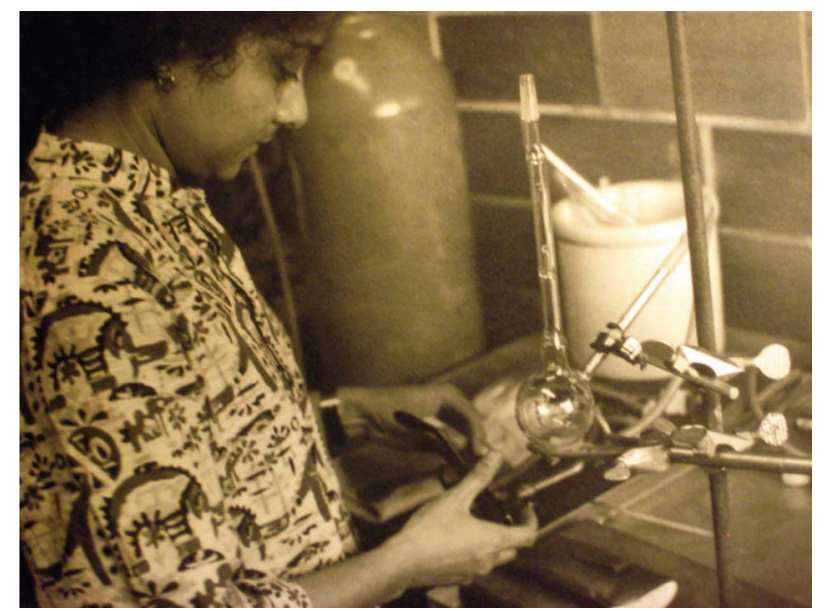

Fig. 1 Tayyaba Hasan performing early organic chemistry work, c. 1985 . and then moved to the Harvard Medical School as a research associate in dermatology, where she worked on tetracycline photosensitization and introduced many of the early concepts in intracellular site targeting, such as the mitochondria of cells. She worked with Alan Oseroff, MD, PhD, and others to demonstrate the concept of antibody conjugate photosensitization of cells for receptor-based photolysis. Her key papers were published in the Proceedings of the National Academy of Science (PNAS), among others.

At MGH, all of Prof. Hasan's career was spent with a primary appointment in the dermatology department, where she met and collaborated with many young research-minded dermatologists and developed long-standing collaborations with many of them. One of these was Christopher Shea, MD, currently Chief of Dermatology at University of Chicago, who met Assistant Professor Tayyaba Hasan in 1984 at MGH through their shared office at Wellman, which was so small that when anyone came in or out of the door he had to stand up to make room. Chris was working with another faculty member trying to target mitochondria by incubating cells with the supravital dye Janus green B and irradiating them with a Q-switched ruby laser, which was not going well because (a) the dye was toxic, and (b) the laser was operated at such high fluences that uncontrollable nonlinear effects dominated. He switched to Prof. Hasan's lab and soon performed the first experiment on cells preloaded with doxycycline. This experiment showed, by the cell-altered pattern of rhodamine-123 localization, that they had sustained highly specific damage to their mitochondria. This study was one of the first proofs of principle for the targeting of subcellular structures by photosensitization, resulting in key papers in Cancer Research and PNAS. Dr. Shea recalls, "Looking back, I am struck by how adventurous and creative Tayyaba was in this effort, to take on a whole new area of investigation, and how generous she was in helping to train me when

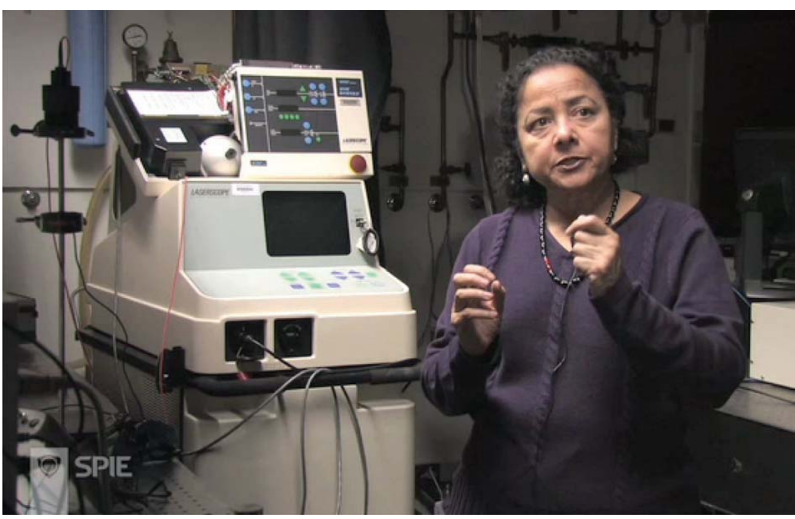

Fig. 2 Tayyaba Hasan explaining photodynamic therapy with clinical laser systems in an interview for the SPIE Newsroom. Watch the interview at http://spie.org/x39658.xml. 


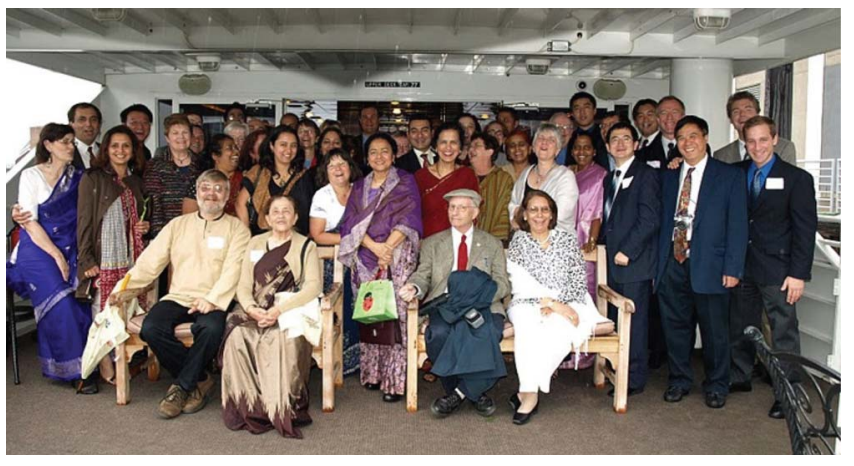

Fig. 3 Tayyaba Hasan at her career award celebratory boat cruise in the Boston Harbor with friends and colleagues from around the world, 2009.

I desperately needed a mentor ... she certainly taught me much more: about the need for rigor in experiments; for courage in daring to go into novel areas and techniques of investigation; and for maintaining intellectual honesty in all things." Another dermatologist, Bernhard Ortel, MD, worked with Prof. Hasan for over a decade focusing on cellularmolecular effects of aminolevulinic acid (ALA) and photodynamic therapy (PDT). Bernhard says that his time of collaboration with Prof. Hasan "...peaked in the exciting development of a program project grant that was first funded in 2001. During this time I was most impressed with her energy, personal effort and continuous commitment to excellence and uncompromising integrity as scientist, leader, and as a loyal colleague. Her leadership keeps benefiting people with diverse backgrounds and with a range of provenances that rivals the United Nations. Tayyaba has the ability to bring the best out of people that work with her and to create an environment where everyone feels happy to contribute and driven to excel. Tayyaba's creative and innovative mind has been the strongest motor of her group's scientific work."

Prof. Hasan spent much of the next phase of her career doing various forms of basic and translational research in many different disease sites beyond dermatology. Her focus was on analysis and innovation around problems that would benefit from PDT. She reported on the early observations of vascular permeability from PDT and development of delivery moieties for optimal uptake in target diseased tissues. Prof. Hasan trained at least as many MD fellows as PhD candidates and postdoctoral fellows. Not only dermatologists, but ophthalmologists, urologists, gynecologists, ear, nose, and throat, and orthopedic surgeons benefited from her mentorship and contributed to the breadth of her success in applied research efforts. Not many researchers inside or outside of the PDT field would be able to show as many clinical applications resulting from their research and development efforts as Prof. Hasan can. In collaboration with the gynecology department, she studied endometrial ablation with PDT and ovarian cancer. She pioneered key innovations in targeted PDT for novel ovarian cancer therapy, which are currently being translated into clinical trials. This is also a good example of her persistence in contributing as much as possible to see the translation of her basic research into clinical trials, even if it takes more than a decade. Her work system- atically shifted away from first-generation photosensitizer use toward ALA for dermatology and basic biology studies, and then to use of benzoporphryin derivative (BPD) for solid tumor and conjugate development. She pioneered the use of BPD in many applications, and the most innovative was the landmark study showing that BPD could be used for elimination of subretinal vessels. This discovery, working with Ursula Schmidt and others, was the foundation for the commercially successful treatment for age-related macular degeneration called Visudyne ${ }^{\circledR}$. This treatment was highly successful and is presently available in 77 countries for the treatment of classic subfoveal choroidal neovascularization that is present in age-related macular generation.

Other key innovations from Prof. Hasan's laboratory include evidence that PDT applications could be used for wound healing, treatment of rheumatoid arthritis, and pathogen and antimicrobial targeting, among other uses. She holds more than 17 patents in these areas and more than 160 peer-reviewed publications, with many in leading medical journals. She continues to direct a long-standing National Cancer Institute Program Project grant in the area of molecular response and imaging-based combination strategies for optimal PDT, which brings together basic scientist and research physicians to work on pancreas cancer and skin cancer treatment design. She also leads three major collaborative grants to develop aptamer targeting conjugates and nanocell-based targeting for ovarian cancer and glioma treatment. Her ability to innovate, inspire, and lead teams of researchers has grown to the point where her work now extends beyond her own research group. She is engaged in collaborations with researchers at leading international institutions, including the Massachusetts Institute of Technology, Harvard, University of Pennsylvania, Cleveland Clinic, Dartmouth, Brooklyn College, Catholic University of America, University of Texas, University College London, University Clinic Ulm, Bar-Ilan University, Swiss Federal Institute of Technology in Lausanne, and Osaka University.

Prof. Hasan has directly trained more than 100 research associates and graduate students, and she maintains guidance over hundreds of young and old scientists at MGH through the Office for Research Career Development. Her impact on biomedical research is incalculable at this point, and her direct footprint in the biomedical optics world is large and continues to grow every day.

Brian W. Pogue

Dartmouth College

Georges A. Wagnieres

Ecole Polytechnique Federale de Lausanne

Lothar D. Lilge

Ontario Cancer Institute

Special Section Guest Editors 